Article

\title{
Ocean Wind Retrieval Models for RADARSAT Constellation Mission Compact Polarimetry SAR
}

\author{
Tianqi Sun ${ }^{1,2}$, Guosheng Zhang ${ }^{2,3} * \mathbb{C}$, William Perrie ${ }^{2}$, Biao Zhang ${ }^{3} \mathbb{C}$, Changlong Guan ${ }^{1}$, \\ Shahid Khurshid ${ }^{4}$, Kerri Warner ${ }^{4}$ and Jian Sun ${ }^{1}$ (D) \\ 1 College of Oceanic and Atmospheric Sciences, Ocean University of China, Qingdao 266100, China; \\ tianqisun.ocean@yahoo.com (T.S.); clguan@ouc.edu.cn (C.G.); sunjian77@ouc.edu.cn (J.S.) \\ 2 Fisheries and Oceans Canada, Bedford Institute of Oceanography, Dartmouth, NS B2Y 4A2, Canada; \\ william.perrie@dfo-mpo.gc.ca \\ 3 School of Marine Sciences, Nanjing University of Information Science and Technology, Nanjing 210044, \\ China; zhangbiao@nuist.edu.cn \\ 4 Earth Applied Science \& Development Section Observation \& Geomatics, Meteorological Service of Canada, \\ Environment and Climate Change Canada, Ottawa, ON K1A 0H3, Canada; \\ shahid.khurshid@canada.ca (S.K.); kerri.warner@canada.ca (K.W.) \\ * Correspondence: zgsheng001@gmail.com; Tel.: +1-902-426-7797
}

Received: 30 October 2018; Accepted: 29 November 2018; Published: 2 December 2018

check for updates

\begin{abstract}
We propose two new ocean wind retrieval models for right circular-vertical (RV) and right circular-horizontal $(\mathrm{RH})$ polarizations respectively from the compact-polarimetry $(\mathrm{CP})$ mode of the RADARSAT Constellation Mission (RCM), which is scheduled to be launched in 2019. For compact RV-polarization (right circular transmit and vertical receive), we build the wind retrieval model (denoted CoVe-Pol model) by employing the geophysical model function (GMF) framework and a sensitivity analysis. For compact RH polarization (right circular transmit and horizontal receive), we build the wind retrieval model (denoted the CoHo-Pol model) by using a quadratic function to describe the relationship between wind speed and $\mathrm{RH}$-polarized normalized radar cross-sections (NRCSs) along with radar incidence angles. The parameters of the two retrieval models are derived from a database including wind vectors measured by in situ National Data Buoy Center (NDBC) buoys and simulated RV- and RH-polarized NRCSs and incidence angles. The RV- and RH-polarized NRCSs are generated by a RCM simulator using C-band RADARSAT-2 quad-polarized synthetic aperture radar (SAR) images. Our results show that the two new RCM CP models, CoVe-Pol and CoHo-POL, can provide efficient methodologies for wind retrieval.
\end{abstract}

Keywords: compact polarization (CP); RADARSAT Constellation Mission (RCM); geophysical model function (GMF); wind retrieval; CoVe-Pol and CoHo-Pol models; right circular horizontal polarization model; right circular vertical polarization model

\section{Introduction}

The Canadian RADARSAT Constellation Mission (RCM) is scheduled for launch in early 2019 and will provide Compact-Polarimetry (CP) synthetic aperture radar (SAR) data. RCM is the evolution of the RADARSAT Program and the successor of RADARSAT-2, which is a satellite constellation carrying three identical C-band SAR satellites. The RCM CP mode consists of a right hand circular transmit and linear/circular receive radar signal, namely right circular-vertical (RV) polarization, right circular-horizontal $(\mathrm{RH})$ polarization, right circular-right circular (RR) polarization and right circular-left circular (RL) polarization. The CP configuration is designed for Earth observation; compared with conventional linear dual-polarization SAR, compact polarimetry SAR can obtain abundant high-resolution information with wider swath [1]. 
Researchers have shown that CP SAR is an efficient imaging mode for ocean surface observation and, therefore, elucidation of algorithms and models for retrieval of ocean surface features and marine variables like wind is critically important [2]. Compact polarization SAR is a dual-polarization radar system which transmits circular (or $45^{\circ}$ ) components and receives two orthogonal polarization components $(\mathrm{V}$ or $\mathrm{H})$ with relative phase $[3,4]$. Accurate information of ocean surface can be obtained from C-band radar data. The main process of physical mechanism is the interactions between the microwaves and related surface water waves. The major interaction is denoted as Bragg scattering when the wavelengths of ocean surface waves are of the same order of radar wavelength. For practical application, wind retrievals from SAR images can be accomplished by C-band geophysical model functions (GMFs), because radar measurements are sensitive to the ocean-surface roughness which is determined by the surface wind field [5]. The GMF is a set of functions between wind vectors and radar backscatter signals denoted as the normalized radar cross section (NRCSs) including dependence on the radar incidence angles.

The C-band GMF model series (CMOD4, CMOD5 and CMOD5.N [5,6]) are used for vertical-vertical (VV) polarization data. For horizontal-horizontal $(\mathrm{HH})$ polarization, there are generally two ways to achieve ocean wind retrieval: (1) conversion of the HH backscatter to VV by a polarization ratio (PR) which is a ratio of VV-polarized NRCS to HH-polarized NRCS [7], or (2) construction of a new relationship between wind vectors and radar backscatters [8]. In terms of C-band linear cross-polarization (VH and HV) ocean backscatter data, C-2PO and C-3PO models are available for wind retrieval $[9,10]$. For wind retrieval from CP SAR mode, recent studies have attempted to retrieve wind speed by converting right circular-vertical (RV) polarization data into linear dual-polarization data, taking advantage of CMOD5 and CMOD5.N models [11,12]. However, the configurations for RV and VV polarizations are different and the CMOD model series were originally developed for C-Band VV-polarized scatterometers rather than SARs. In view of this situation, we propose two new models in this paper to retrieve wind from C-band RV-pol and RH-pol measurements.

CMOD is a well-behaved parameterization to retrieve wind speed, allowing the NRCS to be dependent on the principal parameters, such as radar incidence angle, relative wind direction and wind speed [5]. However, as there are 28 CMOD coefficients, the process of optimizing the CMOD model to CP data sets to generate a new GMF (for CP SAR) is not feasible using normal computing clusters, because of the requirement to adjust such large number of CMOD coefficients to represent the $\mathrm{CP}$ parameters. In order to avoid excessive computation, a sensitivity analysis of the coefficients can play a significant role in optimizing the generation of a new GMF for RV-polarization. The sensitivity analysis is a method to adjust the models by changing the coefficients within a specific range of variations in order to optimize the parameterization and simplify the computation [13,14]. Moreover, another common method to generate a practical empirical algorithm is to fit a function relating the $\mathrm{CP}$ variables and the wind parameters. In this study, we utilize these two methods to construct the wind speed retrieval models for RV- and RH-polarization data.

RADARSAT-2 is beyond its 7-year design life, as it was launched in 2007. Thus the launch of RCM is necessary, and application of CP SAR is a new application offering the advantages of full polarimetry SAR mode, with the possibility of better wide-swath coverage. Based on the simulated CP parameters, this study is a preparation for possible ocean wind retrievals from RCM which will be available in the next year. The remainder of this paper is organized as follows: Section 2 describes the database consisting of CP SAR NRCSs simulated by the RCM simulator using quad-polarized RADARSAT-2 SAR images, and collocated wind vectors observed by NCBC buoys. A new CMOD function for RV-polarization data is proposed based on a sensitivity analysis [13], followed by a performance evaluation, and we present a new model for RH polarization wind speed retrieval. Results and the validations of the two new models are shown in Section 3. Discussion and conclusions are given in Sections 4 and 5, respectively. 


\section{Materials and Methods}

In this section, two wind retrieval models are developed based on the collocated data sets. For the compact RV polarization, we employ the CMOD framework to derive a new GMF by optimizing each coefficient. The resultant formulation is denoted the CoVe-Pol model. In this derivation, sensitivity analysis is used to avoid unnecessarily huge calculations [13]. Through the sensitivity analysis, the computation efficiency of the process to generate the new GMF generation is increased by more the $10^{20}$ times and the accuracy of computed NRCS reaches $10^{-2}$. For the compact RH polarization, we propose the $\mathrm{CoHo}-\mathrm{Pol}$ wind retrieval model which we derive by using a quadratic regression function.

\subsection{Datasets}

The CP parameters were simulated from RADARSAT-2 quad-polarized data using the RCM simulator, which is provided by the Canadian Space Agency [12]. To develop two new wind retrieval models for RV- and RH-polarization data, we build a database consisting of simulated RV- and RH-polarized NRCSs from RADARSAT-2 fine quad-pol SAR images and collocated wind vectors measured by in situ buoys. The RADARSAT-2 quad-pol SAR images over the selected buoys are acquired. Then, these RADARSAT-2 images are converted to RCM CP mode SAR images by using the $\mathrm{CP}$ simulator. Finally, wind speed measured by buoy and the collocated simulated NRCS are paired. The distributions of NRCSs and wind speeds are shown in Figure 1.
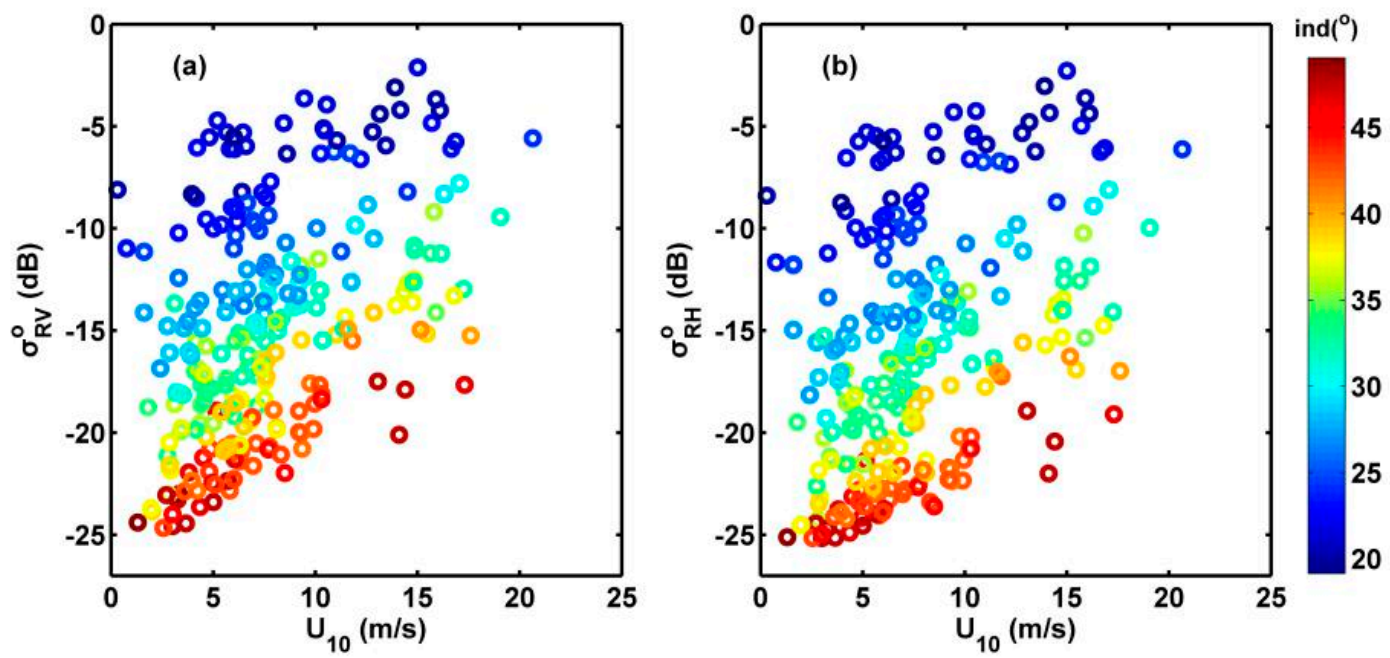

Figure 1. Simulated compact-polarimetry (CP)-polarized normalized radar cross-sections (NRCSs) vs. in situ buoy-measured $U_{10}$ : (a) right circular-vertical (RV) polarization; (b) right circular-horizontal (RH) polarization.

In this database, we have 267 RADARSAT-2 fine quad-pol SAR images, which are processed by a RCM CP simulator to re-construct CP mode images [1]. The results of simulated CP configurations are used as "ground truth" in this study. The RADARSAT Constellation Mission has several polarization configurations: linear mono-polarized, dual linear-polarized (HH/HV, VV/VH, or VV/HH); dual circular transmit-linear receive; and fully polarimetric [12]. The compact polarimetry SAR mode provides four polarimetric datasets, which are $\mathrm{RV}, \mathrm{RH}$, right circular transmit and right circular receive (RR) and right circular transmit and left circular receive (RL). In the medium resolution mode, the pixel spacings in azimuth and range directions are about $100 \mathrm{~m}$ and the associated noise floor is about $-25 \mathrm{~dB}$ [15]. Data from eight National Data Buoy Center (NDBC) buoys collocated with the SAR data are collected, at locations off the east and west coasts of Canada [16]. The buoy locations are shown in Figure 2. At each buoy, the mean wind speed (at $10 \mathrm{~m}$ reference height, hereafter $U_{10}$ ) and direction are measured by two sensors, averaged over 8-min periods and reported hourly. The wind 
speeds observed by the buoys are converted to winds at $10 \mathrm{~m}$ reference height above the ocean surface using the Tropical Ocean and Global Atmosphere Coupled Ocean-Atmosphere Response Experiment (TOGA COARE) bulk flux algorithm [17], and the winds can be considered as neutral winds [6,18]. The temporal separation between the SAR data and the buoy data is restricted to less than $30 \mathrm{~min}$ [11]. The distribution of months is shown in Figure 3. As the SAR is active microwave, the effects of weather and seasons are both almost negligible.

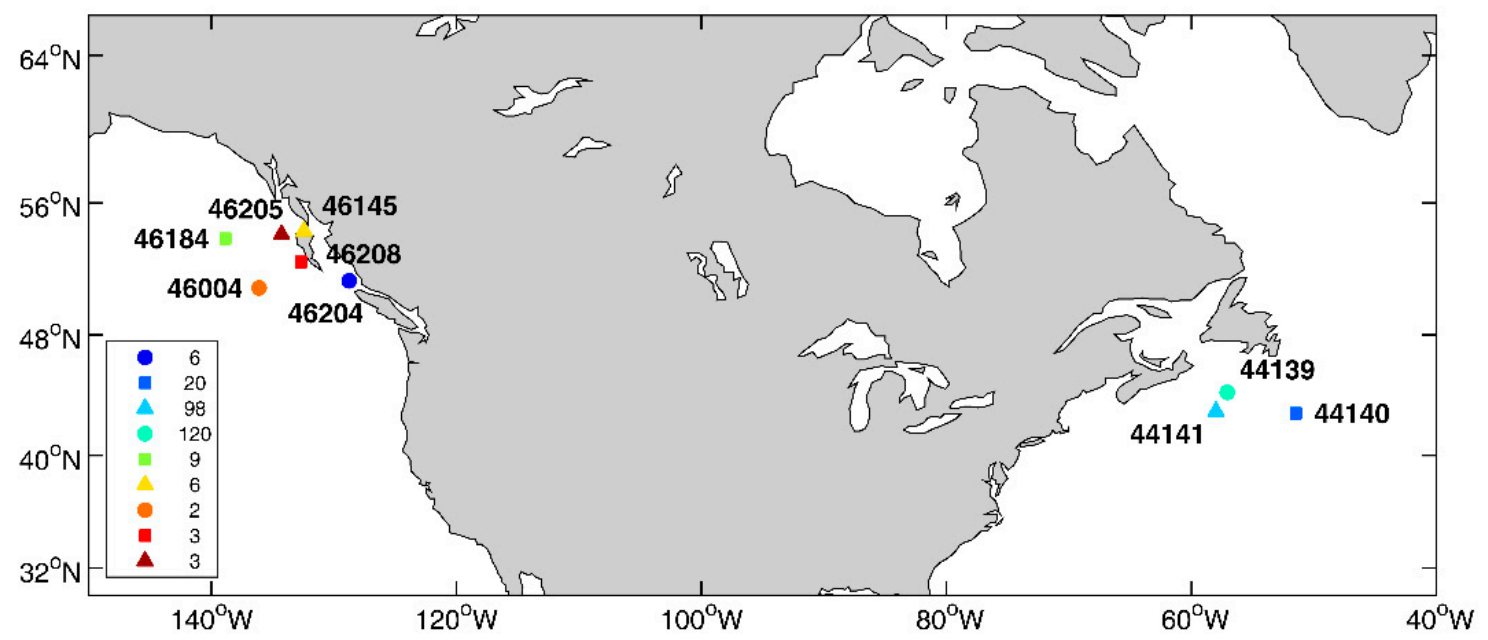

Figure 2. Distribution of buoy locations used in this study. We indicate the number of synthetic aperture radar (SAR) images overlaying the respective buoys in the legend.

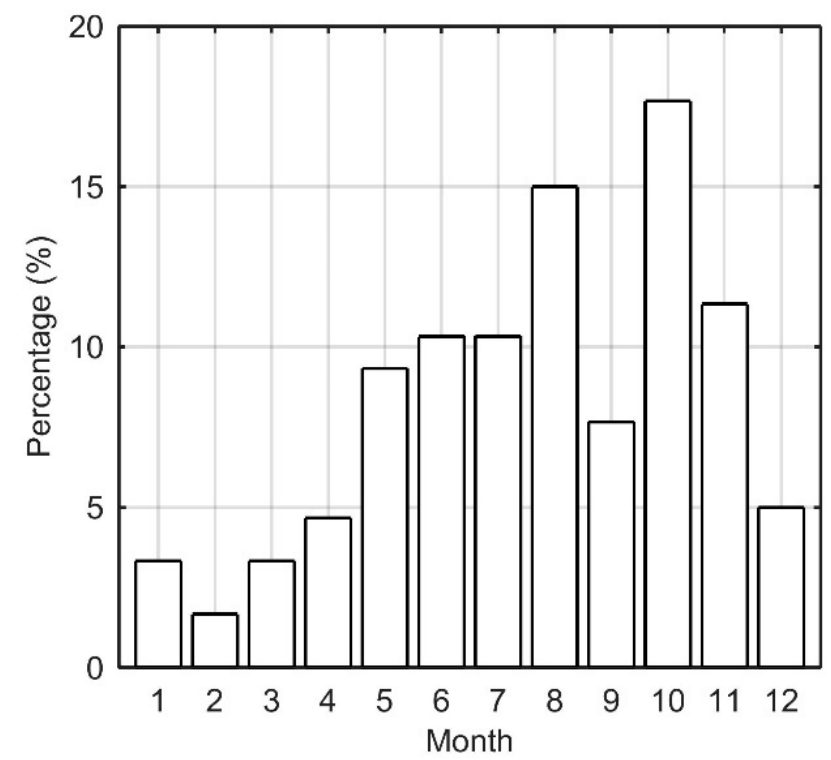

Figure 3. Distribution of months of the collocated data used in this study.

We divide the collected data randomly into two data groups ( $2 / 3$ and $1 / 3$ of the total number). One contains 178 data which are used to generate the new models, and the other contains 89 data, reserved for model testing.

\subsection{CoVe-Pol Model for Right Circular-Vertical (RV) Polarization}

Figure 4 shows the flowchart for the derivation process for the CoVe-Pol model for RV polarization data. As with all GMF functions, an empirical functional relation is used to establish the dependency 
of the normalized backscatter on wind speed, wind direction, and the incidence angle [5]. The general form of the CMOD function is summarized as:

$$
\begin{aligned}
& \sigma^{0}\left(\theta, U_{10}, \varphi\right)= \\
& B 0\left(c_{0}, U_{10}, \theta\right)\left[1+B 1\left(c_{1}, U_{10}, \theta\right) \cos (\varphi)+B 2\left(c_{2}, U_{10}, \theta\right) \cos (2 \varphi)\right]^{1.6}
\end{aligned}
$$

where $\sigma^{0}$ is the NRCS in linear units, $\varphi$ is the relative wind direction, which is the angle between local wind direction and radar look direction (both relative to north), $U_{10}$ is the statistically neutral wind referenced to $10 \mathrm{~m}$ height, $\theta$ is the incidence angle and $B 0, B 1, B 2$ are coefficients depending on $U_{10}, \theta$, the radar frequency and polarization. The dominant term, $B 0$, sets the speed scale for a given measurement. The upwind-crosswind asymmetry term $B 2$ allows for a determination of the wind direction, and $B 1$ is used to resolve the remaining $180^{\circ}$ ambiguity in the wind direction. Coefficients $c_{i}$ complete the definition of the terms B0, B1, B2. Detailed expressions are shown in Appendix A.

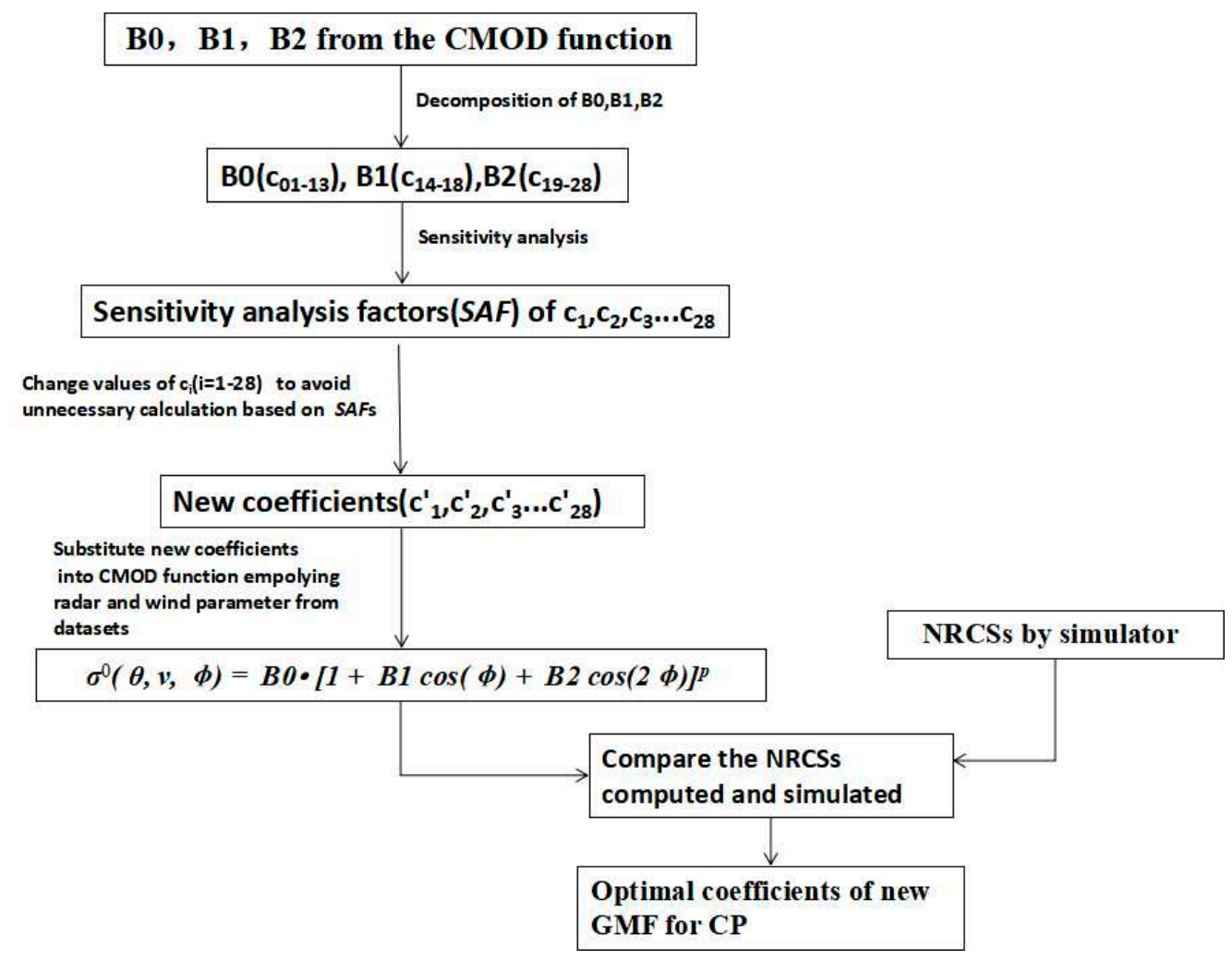

Figure 4. Flowchart for building the CoVe-Pol model through adjustment of the C-band geophysical model function (GMF) model series (CMOD) coefficients obtained by application of a sensitivity analysis.

\subsubsection{Sensitivity Analysis}

As there are $28 \mathrm{CMOD}$ coefficients $\left(c_{1} \sim c_{28}\right)$ in the empirical model formulation, the sensitivity analysis is used to reduce the computations and to allow a determination of the coefficients. We define the sensitivity analysis factor $(S A F)$ of the CMOD coefficients to make the adjustment process more efficient:

$$
S A F=\left|\left(\delta \sigma^{0} / \sigma^{0}\right) /\left(\delta c_{i} / c_{i}\right)\right|
$$

where $\sigma^{0}$ represents the RV-polarized backscatter value (NRCS), the independent variable, and the $c_{i}$ coefficients $\left(c_{1} \sim \mathcal{C}_{28}\right)$ are dependent variables. In this approach, $S A F$ s indicate the degree of influence of each coefficient on the CMOD parameterization. From equation (2), we know that for any particular coefficient $c_{i}$, the corresponding NRCS $\left(\sigma^{0}\right)$ can have multiple values, and in each case the ratio is 
indicative of the degree of influence, $S A F$. If $S A F$ cannot provide the required computational accuracy, the corresponding coefficients $c_{i}$ can be ignored. For any particular coefficient, the basis for this decision is the magnitude of the degree of influence, $S A F$.

Average values of the $S A F s$ under various wind speeds range from $1 \mathrm{~m} / \mathrm{s}$ to $25 \mathrm{~m} / \mathrm{s}$ as shown in Figure 5, assuming typical conditions for radar incidence angles and wind directions. Thus, it is shown that $S A F$ values range widely from 0 to $10^{1}$, which means the influence of different coefficients, $c_{i}$, vary greatly. As shown in Figure 5, very close to 0 , most $S A F$ values are less than 1 and only a few have values exceeding 1 , for three typical radar incidence angles $\left(25^{\circ}, 35^{\circ}\right.$ and $\left.45^{\circ}\right)$. Thus, we only focus on coefficients $c_{i}$, where $S A F$ values indicate greater influence $(>0.1)$ so that the parameterization is simplified and a huge calculation can be avoided.

In this study, we can classify the coefficients by the orders of magnitude of the corresponding $S A F$ s, namely $10^{0}, 10^{-1}$ and less than $10^{-1}$. Thus, when we attempt to get the accuracy of computed NRCS values to $10^{0}$ order of magnitude, we don't need to consider the coefficients with SAF values less than $10^{0}$, because their influence is negligible. Likewise, when we focus on the $10^{-1}$ order of magnitude, the coefficients with $S A F$ values lower than $10^{-1}$ can be ignored. Therefore, based on the $S A F$ s of each coefficient, $c_{i}$, we firstly tune the coefficients whose $S A F$ values have orders of magnitude higher than $10^{\circ}$, without considering other coefficients. Secondly, we tune the coefficients whose $S A F$ values have orders of magnitude higher than $10^{-1}$, without considering other coefficients. Then, we tune the coefficients whose SAF values have progressively higher orders of magnitude than $10^{-1}$. The reason for this approach is that coefficients whose $S A F$ values have orders of magnitude lower than $10^{-1}$ have essentially no effect on computed NRCSs derived from coefficients whose SAF values have higher orders of magnitude.

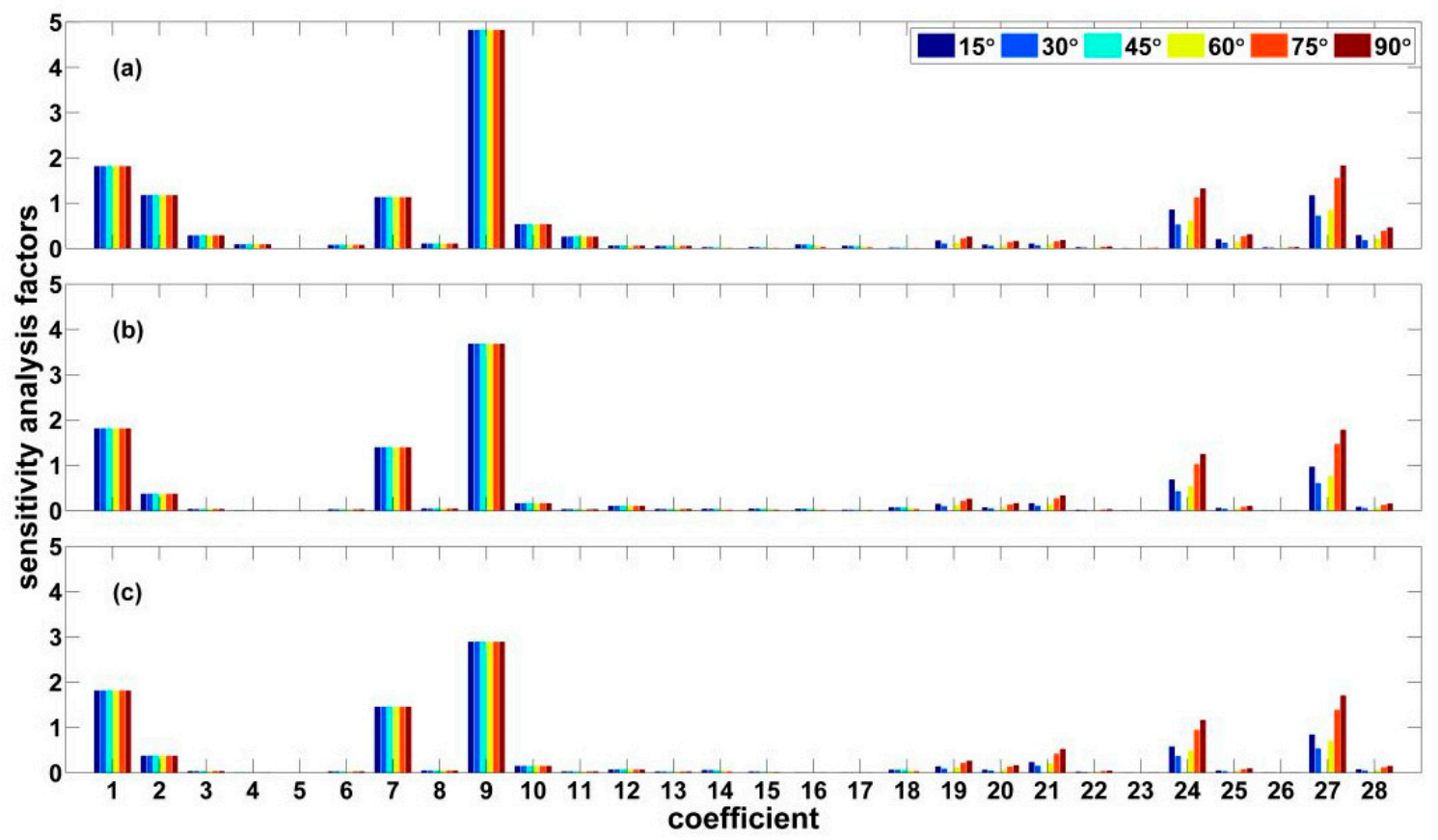

Figure 5. The mean sensitivity factors vs. coefficients: (a) radar incidence angle is 25 degree; (b) radar incidence angle is 35 degree; (c) radar incidence angle is 45 degree. The colors represent different wind directions $\left(15^{\circ}, 30^{\circ}, 45^{\circ}, 60^{\circ}, 75^{\circ}, 90^{\circ}\right)$.

We note that although the radar incidence angles can vary as shown in Figure 5, their influence on the coefficients $c_{i}$, and on the resultant NRCSs is quite minor. Thus, we combine the average SAFs under different radar incidence angles, as shown in Figure 6. The extent of influence for incidence angles on the coefficients $c_{i}$ for the $S A F$ orders of magnitude $10^{0}$ and $10^{-1}$ are shown in Figure $6 \mathrm{a}, \mathrm{b}$, respectively. It is apparent that the coefficients can be divided into three groups according to their 
degrees of influence. The $S A F$ values corresponding to coefficients $c_{1}, c_{7}, c_{9}$ are more than 1 . The $S A F$ values corresponding to coefficients $c_{2}, c_{3}, c_{10}, c_{11}, c_{19}, c_{21}, c_{27}$, and $c_{28}$ are in the range from 0.1 to 1 , and the remaining $c_{i}$ values are under 0.1 . It is notable that the coefficients with higher $S A F$ magnitudes play a part in the adjustment process of the coefficients $c_{i}$, for lower orders of magnitude; but this influence does not work, if we put things the other way around. For example, assuming a $S A F$ value of 1.3219 , the corresponding $c_{i}$ coefficients cannot influence the computed NRCS values above $10^{0}$ order of magnitude, but these $c_{i}$ coefficients do influence NRCS values below $10^{1}$ magnitude such as $10^{0}$ and $10^{-1}$ magnitudes, as reported in this study. Moreover, because $S A F$ values for typical wind directions have similar orders of magnitude, we give the average $S A F$ values for the $c_{i}$ coefficients in Table 1.

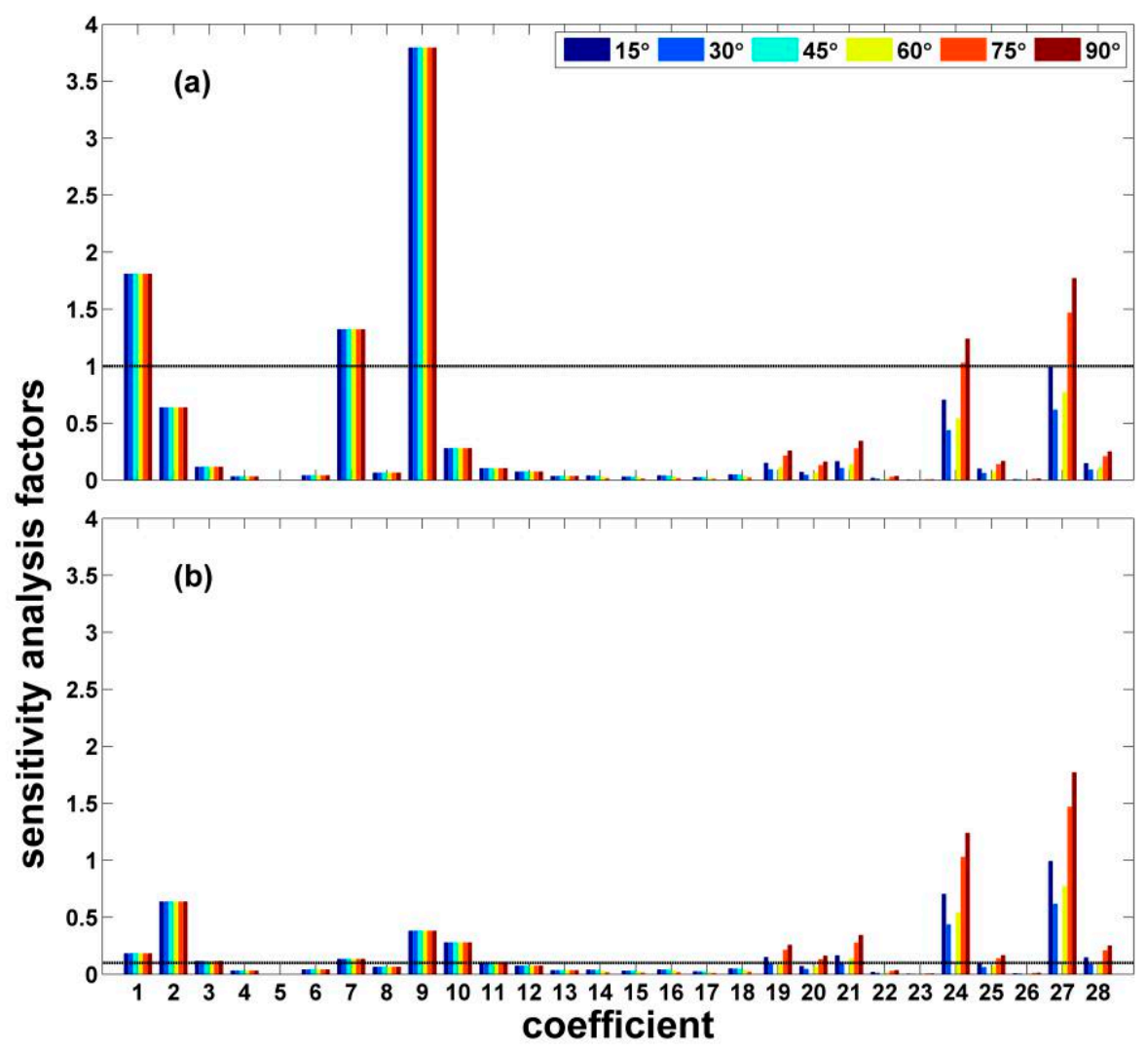

Figure 6. The sensitivity analysis factor $(S A F)$ values for different orders of magnitude for computed NRCSs: (a) SAF over 1; (b) SAF over 0.1. The colors represent different wind directions $\left(15^{\circ}, 30^{\circ}, 45^{\circ}\right.$, $\left.60^{\circ}, 75^{\circ}, 90^{\circ}\right)$

Table 1. Average $S A F$ values of the $c_{i}$ coefficients.

\begin{tabular}{lcllllll}
\hline$S A F$ of $c_{1}$ & 1.8081 & $S A F$ of $c_{8}$ & 0.0630 & $S A F$ of $c_{15}$ & 0.0200 & $S A F$ of $c_{22}$ & 0.0171 \\
$S A F$ of $c_{2}$ & 0.6366 & $S A F$ of $c_{9}$ & 3.7931 & $S A F$ of $c_{16}$ & 0.0276 & $S A F$ of $c_{23}$ & 0.0024 \\
$S A F$ of $c_{3}$ & 0.1145 & $S A F$ of $c_{10}$ & 0.2767 & $S A F$ of $c_{17}$ & 0.0158 & $S A F$ of $c_{24}$ & 0.6617 \\
$S A F$ of $c_{4}$ & 0.0308 & $S A F$ of $c_{11}$ & 0.1012 & $S A F$ of $c_{18}$ & 0.0332 & $S A F$ of $c_{25}$ & 0.0895 \\
$S A F$ of $c_{5}$ & 0 & $S A F$ of $c_{12}$ & 0.0724 & $S A F$ of $c_{19}$ & 0.1381 & $S A F$ of $c_{26}$ & 0.0056 \\
$S A F$ of $c_{6}$ & 0.0399 & SAF of $c_{13}$ & 0.0335 & $S A F$ of $c_{20}$ & 0.0774 & $S A F$ of $c_{27}$ & 0.9411 \\
$S A F$ of $c_{7}$ & 1.3219 & SAF of $c_{14}$ & 0.0262 & $S A F$ of $c_{21}$ & 0.1697 & $S A F$ of $c_{28}$ & 0.1344 \\
\hline
\end{tabular}

\subsubsection{Determination of the Coefficients for CoVe-Pol Model}

We divide the coefficients into three groups according to the magnitude of corresponding $S A F \mathrm{~s}$, which represent the degree of influence of every coefficient $c_{i}$ on the computed NRCSs. Thus, we adjust the coefficients, proceeding from higher magnitudes of their degree of influence, to lower orders of magnitude. Through this method, the computational accuracy of the CoVe model reaches $10^{-2}$, 
and calculation of the required adjustments in the coefficients $c_{i}$ can be achieved using a common computer cluster because the computation has been significantly reduced.

As a first step, we change the values of coefficients $c_{1}, c_{7}, c_{9}$, which determine the accuracy of computed NRCSs at SAF order of magnitude $10^{\circ}$. Thus, the NRCSs are computed by using the conventional GMF formulation with input of the wind speed, incidence angles and wind direction observed by buoys. Comparing the root mean square errors (RMSEs) between the resulting CMOD parameterization and the simulated RCM data (computed and simulated values for NRCSs), there is an optimal set of computed NRCSs with corresponding $c_{1}, c_{7}, c_{9}$ values that minimize the RMSE, as defined in (3) below.

Secondly, in order to achieve the CMOD adjustment at $10^{-1}$ order of magnitude coefficients, we change the values of coefficients $c_{2}, c_{3}, c_{10}, c_{11}, c_{19}, c_{21}, c_{27}, c_{28}$ and $c_{1}, c_{7}, c_{9}$ based on the new CMOD obtained in the first step above. The optimal values of the coefficients are obtained in the same way as previously. For the $10^{-2}$ order of magnitude, there are 25 sensitive coefficients which must be adjusted, making the calculation too huge to be practical. Thus, we tune the $c_{i}$ coefficients empirically. Thus, a GMF with new coefficients for compact RV-polarization SAR is proposed following this adjustment procedure, as displayed in Table A1 in the Appendix A.

The RV-polarized NRCSs computed by the new GMF are in good agreement with simulated RCM data, shown in Figure 7a. Additional details are given in Appendix A.
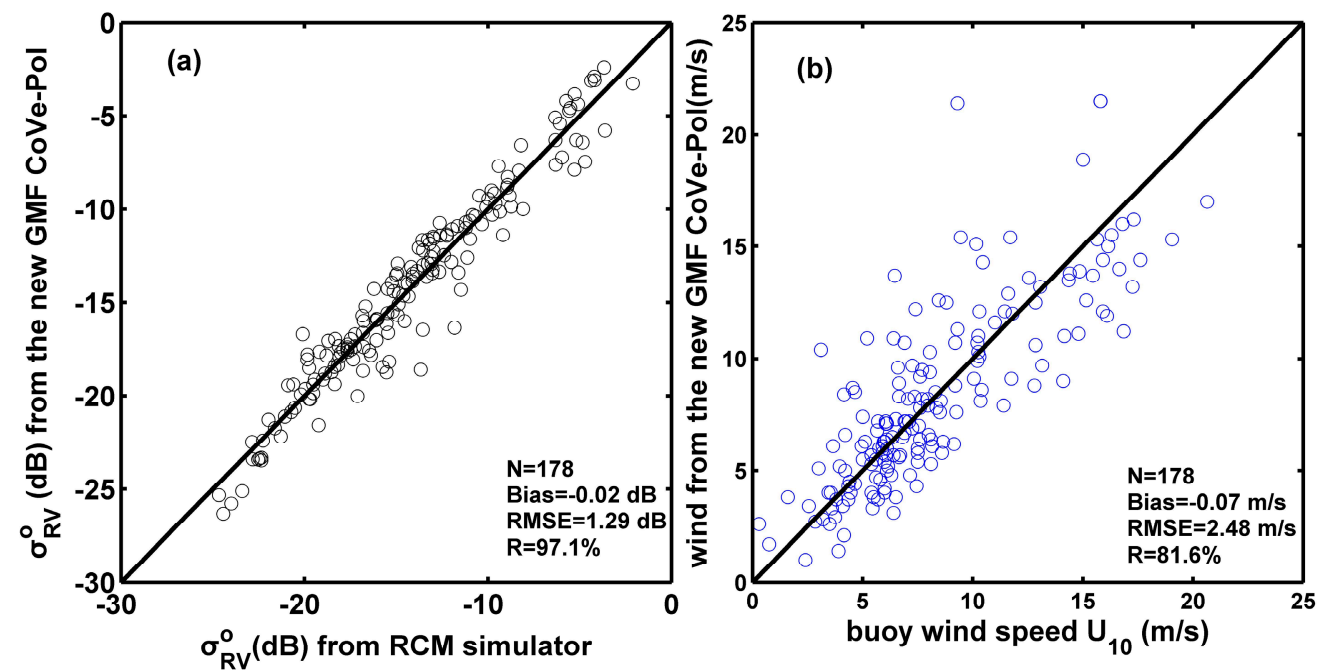

Figure 7. Comparisons of: (a) RV-polarized NRCSs between RADARSAT Constellation Mission (RCM) simulated data and results computed by the new compact RV polarization GMF; and (b) SAR-retrieved wind speeds from CoVe-Pol model and data measured by buoys.

The definitions of bias, RMSE, and correlation coefficient $(R)$ are,

$$
\begin{gathered}
\text { RMSE }=\sqrt{\frac{1}{n} \sum_{i=1}^{n}\left(G_{i}-D_{i}\right)} \\
\text { bias }=\frac{(G-D)}{n} \\
R=\frac{\operatorname{Cov}(G, D)}{\sqrt{\operatorname{Cov}(G, D) \operatorname{Cov}(G, D)}}
\end{gathered}
$$

where $G$ represents the computed results from the GMF, $D$ is the wind speed from the data sets, $n$ is the number of measurements. 


\subsection{CoHo-Pol Model for Right Circular-Horizontal (RH) Polarization}

To build the compact RH polarized wind speed retrieval model, denoted CoHo-Pol, we employ the parameterization method from Komarov et al. [8]. Thus, we use a quadratic relationship in a regression model between buoy wind speed, as a dependent variable, and RH-polarized NRCS, along with incidence angles as independent variables:

$$
V=a_{0}+a_{1} \sigma_{R H}^{0}+a_{2} \theta+a_{3}\left(\sigma_{R H}^{0}\right)^{2}+a_{4} \theta^{2}+a_{5} \sigma_{R H}^{0} \theta
$$

where $V$ is the wind speed (m/s), $\theta$ is the radar incidence angle (degree), and $\sigma_{R H}^{0}$ is the RH-polarized NRCS (dB). Table 2 presents the parameters for the model.

Table 2. Regression coefficients for the horizontal-horizontal (HH) model.

\begin{tabular}{cccccc}
\hline$a_{0}$ & $a_{1}$ & $a_{2}$ & $a_{3}$ & $a_{4}$ & $a_{5}$ \\
\hline-17.8296 & 0.9490 & 1.8640 & 0.0447 & -0.0034 & 0.0525 \\
\hline
\end{tabular}

\subsection{Validation}

As the coefficients CoVe-Pol models and CoHo-Pol models are obtained by training the first group of data sets, additional verification tests are performed using the part of the collected data reserved for model testing. We test the CoVe-Pol model for the NRCS values and wind speed. Thus, we substitute the variables (NRCSs simulated, wind speed observed by buoys, radar incidence angles and wind directions) into the CoVe-Pol model, and we compare the simulated NRCS values with the wind speeds observed by the buoys. To provide additional testing of the $\mathrm{RH}$ model, $\mathrm{CoHo}-\mathrm{Pol}$, the retrieved wind speeds are obtained by substituting $\sigma_{R H}^{0}$ and $\theta$ as given by the reserved data sets into the RH model.

\section{Results}

For wind retrieval, we use simulated compact polarization SAR data and parameters from buoy observations to validate the new RV-polarized GMF, CoVe-Pol. Thus, the wind speed will be determined after we substitute values for the RV-polarized NRCS, incidence angles and wind directions. We compare the wind speeds retrieved by the CoVe-Pol model with the wind speeds observed by buoys in Figure $7 \mathrm{~b}$. The bias is $0.07 \mathrm{~m} / \mathrm{s}$, the RMSE is $2.48 \mathrm{~m} / \mathrm{s}$ and the correlation coefficient is $81.3 \%$. Although the accuracy of CoVe-Pol model appears encouraging, additional tests and validation are still needed in the future, when RCM data is available.

The performance of the regression model for compact RH polarization data, CoHo-Pol, is shown in Figure 8, which indicates that the model is an effective methodology for wind retrieval from $\mathrm{RH}$ polarized data. Comparing model results to wind speeds observed by buoys, the RMSE is $2.37 \mathrm{~m} / \mathrm{s}$.

The wind retrieval models for RV and RH data are presented for compact polarimetry measurements. We test CoVe-Pol model for the NRCS values and wind speed, in Figure 9a,b. The RMSE for $\sigma_{R V}^{0}$ is only $1.28 \mathrm{~dB}$ and for wind speed, $2.36 \mathrm{~m} / \mathrm{s}$, and the values of the correlation coefficients are $97.9 \%$ and $82.4 \%$. These results indicate that CoVe-Pol model is a potentially good method for wind retrieval; the computed NRCSs and retrieved winds are in good agreement with the simulated NRCSs and the independently measured buoy winds.

Comparing the retrieved wind speeds and the buoy observations, the RMSE for winds retrieved by the CoHo-Pol model is $2.39 \mathrm{~m} / \mathrm{s}$, and the correlation coefficient is $81.5 \%$, which is shown in Figure 10 .

Validations demonstrate that the CoVe-Pol and CoHo-Pol models are reliable and useful retrieval models for RV and RH polarized SAR data, respectively. 


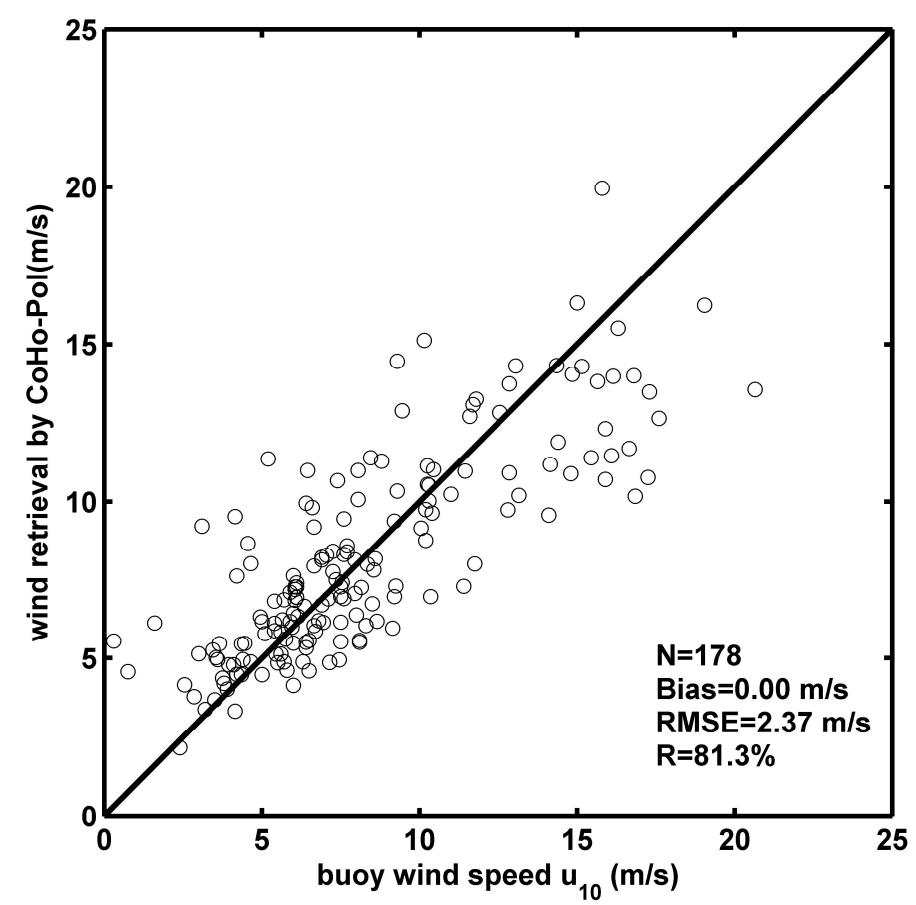

Figure 8. SAR-retrieved wind speeds from RH polarization mode model denoted CoHo-Pol vs. buoy-measured $\mathrm{U}_{10}$ for the reserved training subset.
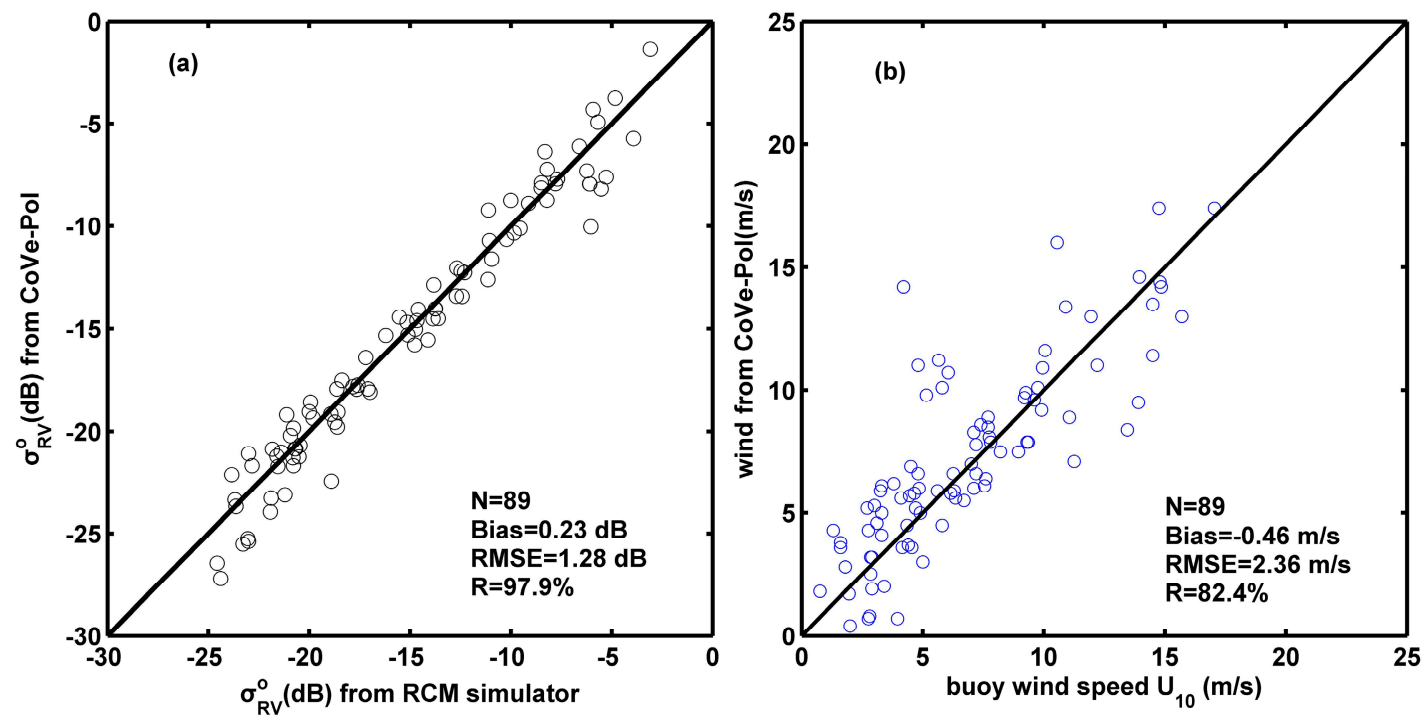

Figure 9. Validation of CoVe-Pol model by inputting wind speed and RV-polarized NRCSs: (a) $\sigma_{R V}^{0}$ from CoVe-Pol model vs. $\sigma_{R V}^{0}$ simulated by the RCM simulator; (b) wind speed retrieved by CoVe-Pol model vs. wind speed measured by buoys. 


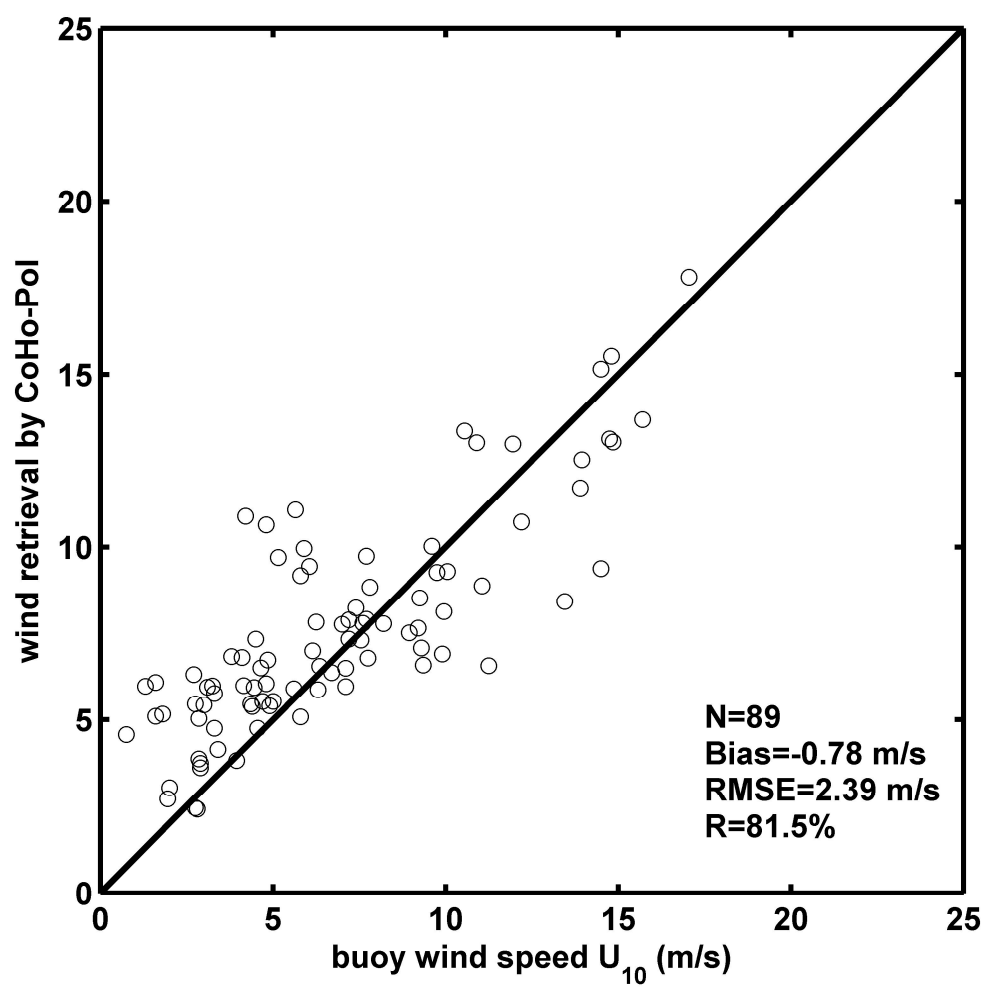

Figure 10. Wind speed retrieved by CoHo-Pol, the RH polarization mode model, vs. reserved wind speeds observed by buoys; the test data subset.

\section{Discussion}

Based on the almost linear relationships between NRCSs of VV and RV polarizations, the C-band RV-polarized wind retrieval model has been simply proposed using the C-band VV-polarized wind retrieval model [11,12]. However, there are two factors should be further discussed: (1) the VV-polarized wind retrieval models, routinely CMODs, are developed for scatterometer but not SAR, and (2) the relationships between the two polarized NRCSs are almost linear but not accurately. Therefore, this study aims to improve the $\mathrm{CP}$ mode wind retrieval accurate by tuning each parameter in the CMOD frame. To reduce the large computations, we employ the sensitivity analysis. Lu et al. (2018) developed a new wind retrieval model for C-band VV-polarization [19]. In the CMOD series, including CMOD4, CMOD5, CMOD5.N, CMOD6, CMOD7 and et al., the equations are the same but with different parameters $[20,21]$. We compare the results from CoVe-Pol models here and the method proposed by Geldsetzer et al. (2015) based on the first data group [11], which is shown in Figure 11. It is obvious that the new model CoVe-POL present better wind retrieval results. 

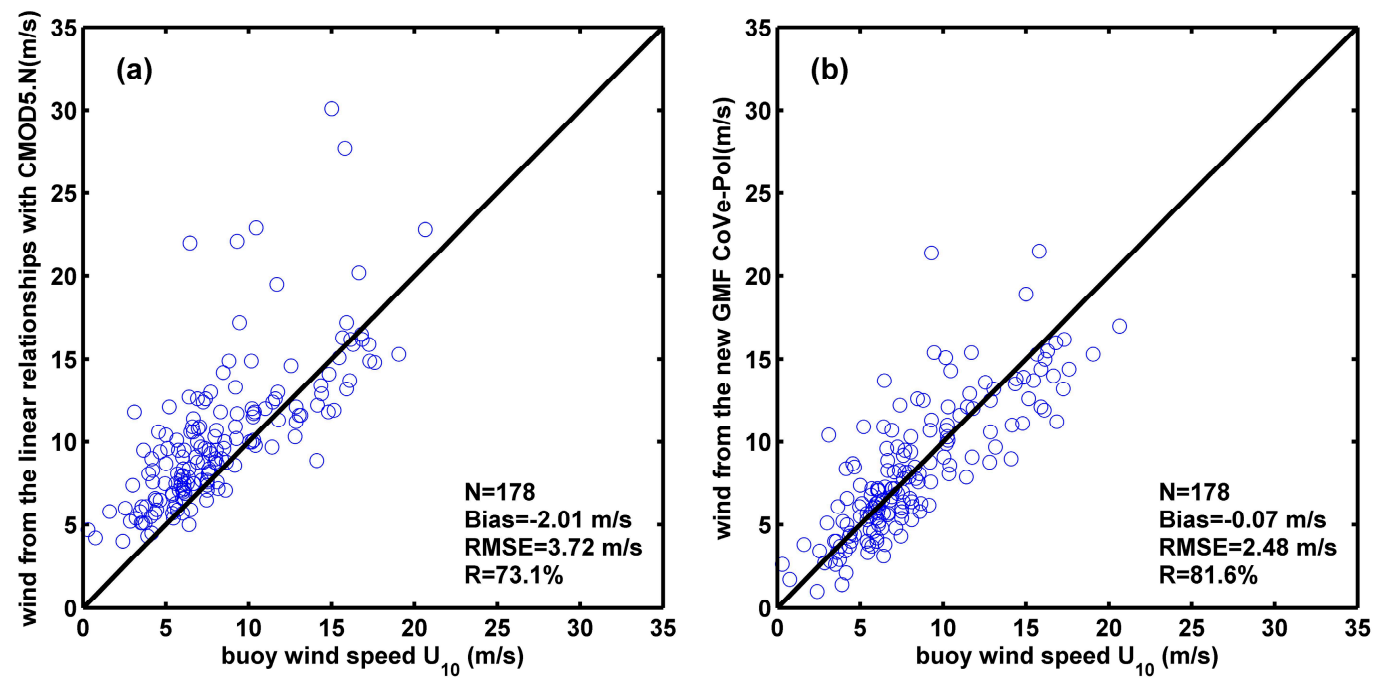

Figure 11. The comparsion of wind speed estimation results between using the linear realtionships with CMOD5.N and using CoVe-Pol model: (a) wind speed estimation through the linear relationships with CMOD5.N vs. wind speed measured by buoys; (b) wind speed retrieved by CoVe-Pol model vs. wind speed measured by buoys.

Zhang et al. (2018) proposed a semi-empirical ocean surface model for RCM CP mode and the simulated results suggest that the RV-polarization has a better potential capability for the ocean wind retrieval than the RH-polarization [22]. This result is consistent with the wind retrieval results here. As indicated by Zhang et al. (2018) [22], the noise floor should be a challenge for sea surface wind retrieval. As shown in Figure 9a, the NRCSs are under-estimated by the new proposed wind retrieval model when the values are around $-25 \mathrm{~dB}$. On one hand, this may be due to the errors of the model. However, on the other hand, this should be caused by the noise floor which is designed as $-25 \mathrm{~dB}$ in the RCM. Therefore, the low wind retrieval from RCM RV and RH polarizations will be a challenge. Moreover, the sea surface ice or oil spill can be detected by SAR due to covering the ocean surface and changing the backscattering features [22]. The determination of oil spill from low wind condition is a current issue from the existing SAR observations. As the noise floor for RADARSAT-2 is much lower than the RCM, this would also be a problem for the sea ice or oil spill study using RCM in the future.

\section{Conclusions}

The estimation of ocean surface winds by SAR is an important research field of satellite remote sensing. Because RCM will provide $\mathrm{CP}$ products which differ from the conventional polarimetry SAR, the establishment of new specific models for potential wind retrieval from CP SAR parameters is an urgent need.

In this paper, we propose two wind retrieval models for C-band RCM SAR CP model: (1) CoVe-Pol model for RV polarization data, and (2) CoHo-Pol model for RH polarization data. The two models are derived from collected data consisting of 267 RADARSAT-2 SAR quad-polarized images and collocated buoy data. These two models can be applied to the real CP data when RCM will have been launched and succeeds in providing data. The CP-polarized data sets are generated from quad-polarized SAR data by a RCM simulator. We have divided these data randomly into two data groups: one for building new models and the other reserved for model testing.

To develop CoVe-Pol model, we carried out a sensitivity analysis in the process of creating the new GMF coefficients. We separate the derivation process for the coefficients into several steps. These steps are designed according to a sequence determined by the orders of magnitude of the CMOD coefficients, in order to reduce the required computations, so that the numerical process can be possible in terms of available computer resources. Utilizing sensitivity analysis factors ( $S A F s$ ) for the coefficients provides an efficient methodology for building a new GMF for the RV polarization data, by adjustment of 
these coefficients. In this approach, when the new GMF is derived, the correlation coefficient between RV-polarized computed NRCSs and simulated NRCSs reaches 97.1\%, and the RMSE is only 1.29 dB. The wind retrieval by the associated CoVe-Pol model parameterization is shown to have a good performance based on the RV polarized SAR data. The RMSE is $2.48 \mathrm{~m} / \mathrm{s}$, and the bias is $0.07 \mathrm{~m} / \mathrm{s}$.

To produce the RH model for wind retrieval, we use a quadratic function in a regression model to relate buoy wind speed to the RH NRCS data along with the radar incidence angles. Comparing results with the winds measured by buoys, the $\mathrm{RH}$ model, denoted CoHo-Pol, is shown to behave well in wind retrievals, with RMSE of $2.37 \mathrm{~m} / \mathrm{s}$. The model results indicate that the $\mathrm{RH}$ model is a useful way to retrieve wind speed as a fast inversion methodology.

We test the two new models, CoVe-Pol and CoHo-Pol, with the reserved test data set, and show that there is strong agreement between both the models and the data. Thus, these two new models can potentially be applied to retrieve wind from CP C-band SAR measurements. In February 2019, the three satellites of RCM are scheduled for launching together. Compared to what we have now with the separated single SAR satellite (e.g., RADARSAT-2 or Sentinel-1), the three continuous observations make the temporal studies of oceanography and/or atmosphere possible. Therefore, the ocean wind retrieval models developed here would be important for temporal oceanography or atmosphere dynamic research based on RCM SAR data.

Author Contributions: Writing-Original Draft Preparation and processing datasets, T.S.; Designing methodology, proposing the model and designing this study, G.Z.; Supervision, writing-Review and editing, W.P.; Validation, B.Z.; Formal Analysis, C.G.; Resources, S.K.; Data Curation, K.W; Software, J.S.

Funding: This research was funded by National Natural Science Youth Foundation of China under Grant 41706193, in part by the Canadian Data Utilization and Application Program "Winds from SAR" RCM Readiness Project between ECCC and DFO, in part by the Canadian Space Agency SWOT and Office of Energy Research and Development (OERD) Programs, and Open Fund of Key Laboratory of Geographic Information Science (Ministry of Education), East China Normal University under Grant No. KLGIS2017A06, National Natural Science Foundation of China under Grant Nos. 41376010), and the Excellent Youth Science Foundation of Jiangsu Province under Grant BK20160090.

Acknowledgments: The authors thank the China Scholarship Council for supporting this scientific research cooperation under the sponsorship [2015] 3022, the Canadian Space Agency for providing RADARSAT-2 data, NOAA NDBC for supplying buoy data (http:/ / www.ndbc.noaa.gov/).

Conflicts of Interest: The authors declared that they have no conflicts of interest to this work.

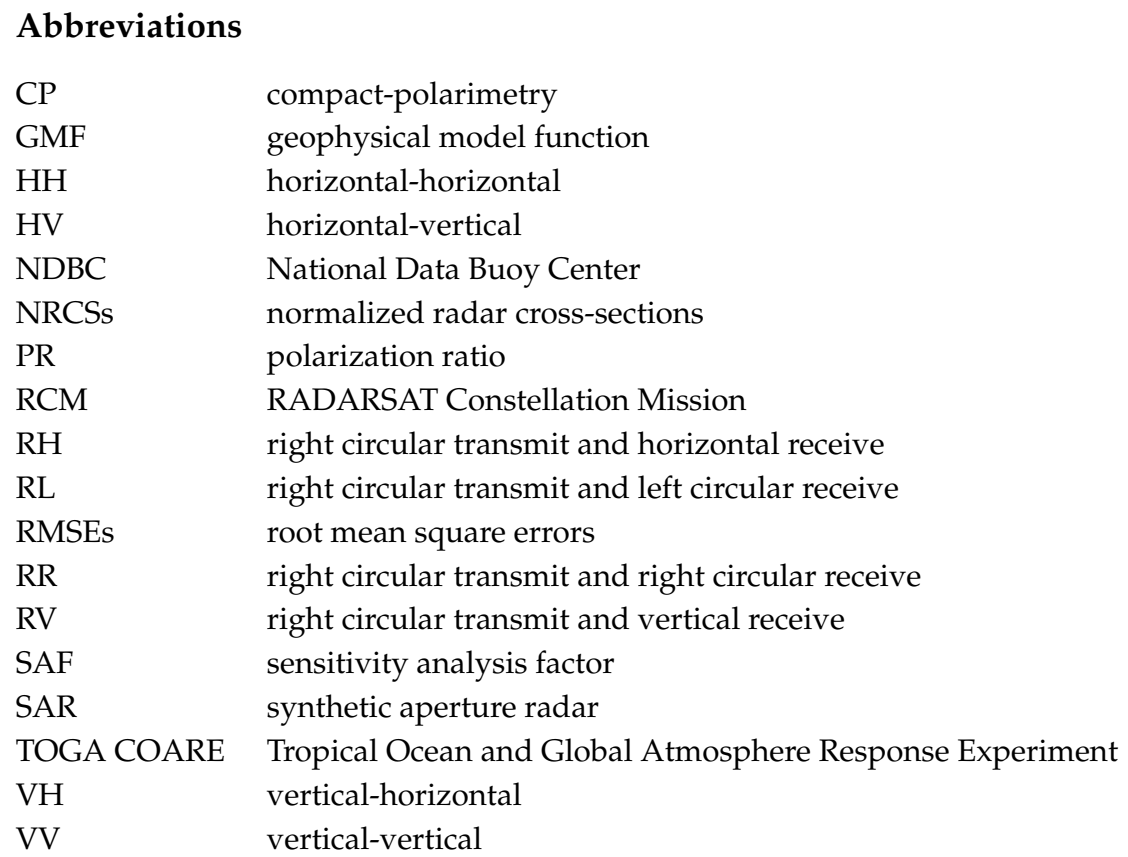




\section{Appendix A Cove-Pol Model Formulation and Coefficients}

The form of the CoVe-Pol model parameterization:

$$
\begin{gathered}
\sigma^{0}\left(\theta, U_{10}, \varphi\right)= \\
=B 0\left(c_{0}, U_{10}, \theta\right)\left[1+B 1\left(c_{1}, U_{10}, \theta\right) \cos (\varphi)+B 2\left(c_{2}, U_{10}, \theta\right) \cos (2 \varphi)\right]^{1.6}
\end{gathered}
$$

where $B 0, B 1$ and $B 2$ are functions of wind speed $U_{10}$ and incidence angle $\theta$, or alternatively, $\chi=(\theta-40) / 25$. The $B 0$ term is defined as:

$$
B 0=10^{a_{0}+a_{1} U_{10}} f\left(a_{2} U_{10}, s_{0}\right)
$$

where,

$$
f\left(s, s_{0}\right)=\left\{\begin{array}{cc}
\left(s_{0}\right)^{\alpha} g\left(s_{0}\right), & s<s_{0} \\
g(s), & s>s_{0}
\end{array}\right.
$$

where,

$$
g(s)=1 /(1+\exp (-s)), \text { and } \alpha=s_{0}\left(1-g\left(s_{0}\right)\right)
$$

The functions $a_{0}, a_{1}, a_{2}, \gamma$ and $s_{0}$ depend on incidence angle only:

$$
\begin{gathered}
a_{0}=c_{1}+c_{2} x+c_{3} x^{2}+c_{4} x^{3} \\
a_{1}=c_{5}+c_{6} x \\
a_{2}=c_{7}+c_{8} x \\
\\
\gamma=c_{9}+c_{10} x+c_{11} x^{2} \\
s_{0}=c_{12}+c_{13} x
\end{gathered}
$$

The $B 1$ term is modeled as follows:

$$
B 1=\frac{c_{14}(1+x)-c_{15} v\left(0.5+x-\tanh \left[4\left(x+c_{16}+c_{17} v\right)\right]\right)}{1+\exp \left(0.34\left(v-c_{18}\right)\right)}
$$

The $B 2$ term was chosen as,

$$
B 2=\left(-d_{1}+d_{2} v_{2}\right) \exp \left(-v_{2}\right)
$$

Here $v_{2}$ is given by,

$$
v_{2}=\left\{\begin{array}{cc}
a+b(y-1)^{n}, & y<y_{0} \\
y & , y \geq y_{0} \\
y=\frac{v+v_{0}}{v_{0}} &
\end{array}\right.
$$

where,

$$
\begin{gathered}
y_{0}=c_{19}, \quad n=c_{20} \\
a=y_{0}-\left(y_{0}-1\right) / n, \quad b=1 /\left[n\left(y_{0}-1\right)^{n-1}\right]
\end{gathered}
$$

The quantities $v_{0}, d_{1}$ and $d_{2}$ are functions of incidence angle only,

$$
\begin{gathered}
v_{0}=c_{21}+c_{22} x+c_{23} x^{2} \\
d_{1}=c_{24}+c_{25} x+c_{26} x^{2} \\
d_{2}=c_{27}+c_{28} x
\end{gathered}
$$

The coefficients are given in Table A1.

Table A1. CoVe-Pol coefficients.

\begin{tabular}{cccccccc}
\hline$c_{\mathbf{1}}$ & -0.9200 & $c_{\mathbf{8}}$ & 0.0159 & $c_{\mathbf{1 5}}$ & 0.0064 & $c_{\mathbf{2 2}}$ & -3.2592 \\
$c_{\mathbf{2}}$ & -1.1935 & $c_{\mathbf{9}}$ & 5.4536 & $c_{\mathbf{1 6}}$ & 0.3141 & $c_{\mathbf{2 3}}$ & 1.2905 \\
$c_{\mathbf{3}}$ & 0.0321 & $c_{\mathbf{1 0}}$ & 0.2633 & $c_{\mathbf{1 7}}$ & 0.0117 & $c_{\mathbf{2 4}}$ & 6.0876 \\
$c_{\mathbf{4}}$ & 0.3421 & $c_{\mathbf{1 1}}$ & -2.2313 & $c_{\mathbf{1 8}}$ & 45.4000 & $c_{\mathbf{2 5}}$ & 2.3296 \\
$c_{\mathbf{5}}$ & 0 & $c_{\mathbf{1 2}}$ & 0.0472 & $c_{\mathbf{1 9}}$ & 2.0293 & $\boldsymbol{c}_{\mathbf{2 6}}$ & 0.3168 \\
$c_{\mathbf{6}}$ & 0.0040 & $c_{\mathbf{1 3}}$ & -0.0689 & $c_{\mathbf{2 0}}$ & 2.9350 & $c_{\mathbf{2 7}}$ & 4.0550 \\
$c_{\mathbf{7}}$ & 0.0882 & $c_{\mathbf{1 4}}$ & 0.0043 & $c_{\mathbf{2 1}}$ & 16.7318 & $c_{\mathbf{2 8}}$ & 1.5237 \\
\hline
\end{tabular}




\section{References}

1. Charbonneau, F.T.; Brisco, B.; Raney, R.K.; McNairn, H.; Liu, C.; Vachon, P.W.; Shang, J.; DeAbreu, R.; Champagne, C.; Merzouki, A.; et al. Compact Polarimetry Overview and Applications Assessment. Can. J. Remote Sens. 2010, 36, S298-S315. [CrossRef]

2. Cloude, S.R.; Goodenough, D.G.; Chen, H. Compact Decomposition Theory. IEEE Geosci. Remote Sens. Lett. 2012, 9, 28-32. [CrossRef]

3. Souyris, J.C.; Imbo, P.; Fjortoft, R.; Mingot, S.; Lee, J.S. Compact polarimetry based on symmetry properties of geophysical media: The /spl pi/ 4 mode. IEEE Trans. Geosci. Remote Sens. 2005, 43, 634-646. [CrossRef]

4. Keith, R.K. Hybrid-Polarity SAR Architecture. IEEE Trans. Geosci. Remote Sens. 2007, 45, 3397-3404. [CrossRef]

5. Hersbach, H.; Stoffelen, A.; De Haan, S. An improved C-band scatterometer ocean geophysical model function: CMOD5. J. Geophys. Res. 2007, 112, C03006. [CrossRef]

6. Hersbach, H. Comparison of C-Band Scatterometer CMOD5.N Equivalent Neutral Winds with ECMWF. J. Atmos. Ocean. Technol. 2010, 27, 721-736. [CrossRef]

7. Vachon, P.W.; Dobson, F.W. Wind Retrieval from RADARSAT SAR Images: Selection of a Suitable C-Band HH Polarization Wind Retrieval Model. Can. J. Remote Sens. 2000, 26, 306-313. [CrossRef]

8. Komarov, A.S.; Zabeline, V.; Barber, D.G. Ocean Surface Wind Speed Retrieval from C-band SAR Images Without Wind Direction Input. IEEE Trans. Geosci. Remote Sens. 2013, 52, 980-990. [CrossRef]

9. Zhang, B.; Perrie, W. Cross-Polarized Synthetic Aperture Radar: A New Potential Measurement Technique for Hurricanes. Bull. Am. Meteor. Soc. 2012, 93, 531-541. [CrossRef]

10. Zhang, G.S.; Li, X.F.; Perrie, W.; Hwang, P.A.; Zhang, B.; Yang, X.F. A Hurricane Wind Speed Retrieval Model for C-band RADARSAT-2 Cross-polarization ScanSAR Images. IEEE Trans. Geosci. Remote Sens. 2017, 55, 4766-4774. [CrossRef]

11. Denbina, M.; Collins, M.J. Wind Speed Estimation using C-band compact polarimetric SAR for wide swath imaging modes. ISPRS J. Photogramm. Remote Sens. 2016, 113, 75-85. [CrossRef]

12. Geldsetzer, T.; Charbonneau, F.; Arkett, M.; Zagon, T. Ocean Wind Study Using Simulated RCM Compact-Polarimetry SAR. Can. J. Remote Sens. 2015, 41, 418-430. [CrossRef]

13. Hamby, D.M. A Review of Techniques for Parameter Sensitivity Analysis of Environmental Models. Environ. Monit. Assess. 1994, 32, 135-154. [CrossRef] [PubMed]

14. Holvoet, K.; van Griensven, A.; Seuntjens, P.; Vanrolleghem, P.A. Sensitivity analysis for hydrology and pesticide supply towards the river in SWAT. Phys. Chem. Earth 2005, 30, 518-526. [CrossRef]

15. Canadian Space Agency. Available online: http://www.asc-csa.gc.ca/eng/satellites/radarsat/ (accessed on 20 October 2018).

16. National Data Buoy Center. Available online: http://www.ndbc.noaa.gov (accessed on 20 October 2018).

17. Fairall, C.W.; Bradley, E.F.; Hare, J.E.; Grachev, A.A.; Edson, J.B. Bulk Parameterization of Air-Sea Fluxes: Updates and Verification for the COARE Algorithm. J. Clim. 2003, 16, 571-591. [CrossRef]

18. Smith, S.D. Coefficients for sea surface wind stress, heat flux, and wind profiles as a function of wind speed and temperature. J. Geophys. Res. 1988, 93, 15467-15472. [CrossRef]

19. Lu, Y.R.; Zhang, B.; Perrie, W.; Mouche, A.A.; Li, X.F.; Wang, H. A C-Band Geophysical Model Function for Determining Coastal Wind Speed Using Synthetic Aperture Radar. IEEE J. Sel. Top. Appl. Earth Obs. Remote Sens. 2018, 11, 2417-2428. [CrossRef]

20. Elyouncha, A.; Neyt, X.; Stoffelen, A.; Verspeek, J. Assessment of the corrected CMOD6 GMF using scatterometer data. Remote Sens. Ocean Sea Ice Coast. Waters Large Water Reg. 2015, 9638. [CrossRef]

21. Stoffelen, A.; Verspeek, J.; Vogelzang, J.; Verhoef, A. The CMOD7 Geophysical Model Function for ASCAT and ERS Wind Retrievals. IEEE J. Sel. Top. Appl. Earth Obs. Remote Sens. 2017, 10, 2123-2134. [CrossRef]

22. Zhang, G.S.; Perrie, W.; Zhang, B.; Khurshid, S.; Warner, K. Semi-empirical ocean surface model for compact-polarimetry mode SAR of RADARSAT Constellation Mission. Remote Sens. Environ. 2018, 217, 52-60. [CrossRef]

(C) 2018 by the authors. Licensee MDPI, Basel, Switzerland. This article is an open access article distributed under the terms and conditions of the Creative Commons Attribution (CC BY) license (http:/ / creativecommons.org/licenses/by/4.0/). 